\title{
Study on the use of uninorm aggregation operators in linguistic fuzzy modeling
}

\author{
Juan M. Bardallo, Miguel A. de Vega, Francisco A. Márquez, Antonio Peregrín \\ Department of Information Technologies, \\ University of Huelva, 21819 Huelva, Spain \\ Email: \{alfredo.marquez, peregrin\}@dti.uhu.es.es \\ \{devega.alcantara, bardallo.gonzalez\}@gmail.com
}

\begin{abstract}
This work aims to develop a practical study of the uninorms as rule antecedent aggregation operator family in Linguistic Fuzzy Modeling. Although they are well known from a theoretical point of view, they have only recently been introduced in a few specific and recent applications. Uninorms are parameterized operators that combine the membership values in the antecedent in a more flexible way than the classically employed tnorms. Therefore, we carried out an in-depth experimental study with six of them, using 23 regression problems of different size and complexity, and reached some conclusions.
\end{abstract}

Keywords: Linguistic fuzzy modeling, uninorms, evolutionary fuzzy systems, adaptive inference systems.

\section{Introduction}

The design of Fuzzy Rule based Systems (FRBS) for practical fuzzy modeling is a complex task that requires finding the appropriate Knowledge Base, which in fact usually has greater impact on the behavior.

Nevertheless, we can also deal with other important elements of FRBSs, such as the setup of the Inference System and Defuzzification Interface [1, 2]. The most interesting point is that they are complementary to those based on Knowledge Base improvements, and both together can reach higher levels of quality.

Furthermore, these elements can use parameters and become adaptive [3]. It is acknowledged that improvements in the accuracy of fuzzy modeling using adaptive Inference Systems and a Defuzzification Interface could be highly relevant $[4,5,6]$.

Focusing on the adaptive Inference System, the most common approach is to use adaptive conjunction operators as antecedent aggregation operators [4]. Then, one or more parameters are employed in their expressions to modify their behavior, allowing us to customize the way each rule infers.

However, in the literature there are other suitable antecedent aggregation operators [7, 8, 9, 10]. In this work, we describe and carry out a practical comparative study on the use of more flexible antecedent aggregation operators. i.e. the uninorms family [11] as they have demonstrated their good performance in several applications $[9,10]$, but nowadays, there are no practi- cal studies comparing them within their own family to see which ones shows the best behavior.

Uninorms are parameterized operators that provide more degrees of freedom because they can behave as both, conjunction and disjunction, expanding the antecedent aggregation options and therefore encouraging better cooperation between the fuzzy rules and consequently more accurate fuzzy models. They can provide a better chance of adapting the way in which each rule is involved beyond the usual expression of connective aggregation operator implemented with a t-norm.

To carry out the aforementioned comparative study we chose different representative uninorms of the minimum family $\left(U_{\min }\right)$ [12]. This subfamily fulfils the requirements for use as conjunction operators. We tested them with 23 different problems with diverse number of variables and examples, and perform a nonparametric statistical analysis to establish a comparison among them.

In order to show what we did, the paper is organized as follows: Section 2 describes the uninorms family of operators. Section 3 depicts the evolutionary learning algorithm we propose to learn their parameter, and Section 4 studies and analyzes the practical behavior shown by them. Finally, Section 5 presents some concluding remarks.

\section{Linguistic fuzzy modeling using uninorms as ag- gregation operators.}

In this section, we depict the basics of the uninorms used as fuzzy antecedent aggregation operators in linguistic or Mamdani fuzzy modeling. First of all, we briefly describe the use of aggregation operators in fuzzy modeling, the previous use of parameterized aggregation operators, and finally, we arrive to the use of uninorms.

As is well known, linguistic FRBSs for system modeling use IF - THEN rules of the following type:

$R_{i}$ : If $X_{i l}$ is $A_{i l}$ and $\ldots$ and $X_{i n}$ is $A_{i n}$ then $Y$ is $B_{i}$

with $i=1$ to $\mathrm{M}$, where $\mathrm{M}$ stands for the number of rules of the Rule Base (RB), $X_{i l}$ to $X_{i n}$ and $Y$ for the input and output variables respectively, and $A_{i l}$ to $A_{i n}$ and $B_{i}$ for the involved antecedents and consequent labels, respectively.

The expression of the Compositional Rule of Inference in fuzzy modeling with singleton fuzzification is as follows: 
$\mu_{B^{\prime}}(y)=I\left(A\left(\mu_{A l}\left(x_{1}\right), \ldots, \mu_{A n}\left(x_{n}\right)\right), \mu_{B}(y)\right)$

where $\mu_{B^{\prime}}$ is the membership function of the inferred consequent, $I$ is the rule connective or implication operator, $A$ is the aggregation operator or antecedent connection, $\mu_{A i}$ are the values of the matching degree of each input of the system with the membership functions of the rule antecedents, and $\mu_{\mathrm{B}}$ is the consequent of the rule.

Therefore, the Inference System performs the two following tasks:

1) First, it computes $A\left(\mu_{A I}\left(x_{l}\right), \ldots, \mu_{A n}\left(x_{n}\right)\right)$, which is the aforementioned matching degree of each rule. Usually, the aggregation operator $A$ (.) is modeled with a t-norm.

2) Second, it infers using the fuzzy rule connective $I($.$) , the previously computed matching degree and the$ consequent of the rule. Fuzzy rule connectives can be classified into different families, of which implication functions [13] and t-norms [14] are the best known.

Frequently, t-norms are used as aggregation operator and inference operator $[2,14,15]$. The expressions of the most used classic t-norms are:

$$
\begin{aligned}
& T_{\text {Minimum }}(x, y)=\operatorname{Min}(x, y) \\
& T_{\text {Hamacher }}(x, y)=\frac{x \cdot y}{(x+y-x \cdot y)} \\
& T_{\text {Algebraic }}=x \cdot y \\
& T_{\text {Einstein }}(x, y)=\frac{x \cdot y}{(1+(1-y) \cdot(1-y))} \\
& T_{\text {Bounded }}(x, y)=\operatorname{Max}(0, x+y-1) \\
& T_{\text {Drastic }}(x, y)= \begin{cases}x, & \text { if } y=1 \\
y, & \text { if } x=1 \\
0, & \text { otherwise }\end{cases}
\end{aligned}
$$

Although their use is less common, t-conorms [6] (disjunctive aggregation) can also be used. T-conorms (also called s-norms) are dual to t-norms under the order-reversing operation which assigns $1-\mathrm{x}$ to $\mathrm{x}$ in [0, 1]. Given a t-norm, the complementary t-conorm is defined by:

$$
\perp(a, b)=1-\mathrm{T}(1-a, 1-b)
$$

The most typical, which we shall use later in this study, are:

$$
\begin{aligned}
& S_{\text {Maximun }}(x, y)=\operatorname{Max}(x, y) \\
& S_{\text {Hamacher }}(x, y)=\frac{(x+y-2 x y)}{(1-x \cdot y)} \\
& S_{\text {Algebraic }}=x+y-x \cdot y \\
& S_{\text {Einstein }}(x, y)=\frac{x+y}{(1+x \cdot y)} \\
& S_{\text {Bounded }}(x, y)=\operatorname{Min}(1, x+y)
\end{aligned}
$$

$S_{\text {Drastic }}(x, y)= \begin{cases}x, & \text { if } y=0 \\ y, & \text { if } x=0 \\ 1, & \text { otherwise }\end{cases}$

In another sense, the use of parameterized aggregation of the antecedents in fuzzy modeling to particularize the way each rule aggregates its antecedents has been studied, e.g., in [4, 8]: In [8] an interesting study was carried out, seeking better performance than traditional minimum or product t-norms for the antecedent connections. The authors suggested the use of adaptive t-norms to look for better performance than those not parameterized. They studied the use of adaptive connectors that are extended from t-norms and t-conorms

\begin{tabular}{|c|c|c|c|}
\hline $\begin{array}{c}\text { parametric } \\
\text { t-norm }\end{array}$ & & & $\begin{array}{c}\text { parametric t- } \\
\text { conorm }\end{array}$ \\
\hline S-OWA & t-norm & S-OWA & t-conorm \\
\hline & \multicolumn{2}{|c|}{ compensatory and } & \\
\hline $\begin{array}{l}\text { Minimi } \\
\text { t-norm }\end{array}$ & & & $\begin{array}{c}\text { drastic } \\
\text { sum }\end{array}$ \\
\hline
\end{tabular}
in order to cover the range between them, including SOWA and compensatory and operators and many others. Fig. 1 shows the ranges covered by them. Other recent studies can be found in [7]. In [4] authors compare the use of a single parameter to globally tune the behavior of the antecedent connector and individual parameters for every rule, having a local tuning mechanism of the behavior of the inference system for every rule. This model shows the highest accuracy because of its high degree of freedom. Therefore, in this study, we learn the antecedent connector for every rule separately.

Fig. 1: Ranges covered by adaptive t-norms, adaptive tconorms, compensatory and, and S-OWA operators.

In $[4,5]$, also using adaptive t-norms, the greatest impact of the parameterized aggregation is shown against the parameterization of the inference or rule connective operator.

Furthermore, some authors studied forms of aggregation operators different from conjunction in linguistic fuzzy modeling, as for certain applications other operators performing between the conjunction and disjunction showed good behavior $[11,16]$. One of these is the uninorms [11], a family of operators that have been used quite successfully in various applications $[9,10]$.

Uninorms were introduced by Yager and Rybalov [11] as a generalization of both t-norms and t-conorms. They allow for a neutral element lying anywhere in the unit interval rather than at one or zero, as in the case of t-norms and t-conorms.

Definition [10]. A uninorm $U$ is a commutative, associative and increasing binary operator $U:[0,1]^{2} \rightarrow$ $[0,1]$ with a neutral element

$e \in[0,1]$, i.e., $U(e, x)=x, \forall x \in[0,1]$

Obviously, the case $e=1$ leads back to t-norms, while the case $e=0$ leads back to t-conorms. For any uninorm $U$, one of the following two cases always holds [12]:

- $\quad U$ is a conjunctive (or and-like) uninorm: $U(1,0)$ $=U(0,1)=0$ 
- $\quad U$ is a disjunctive (or or-like) uninorm: $U(1,0)=$ $U(0,1)=1$.

The structure of any uninorm $U$, as shown in Fig. 2, is a t-norm in $[0, e]^{2}$ and a t-conorm in $[e, 1]^{2}$ and takes values between the minimum and maximum in the other cases, that is, in the subset of unit square given for

$D_{e}=[0, e) \times(e, 1] \cup(e, 1] \times[0, e)$

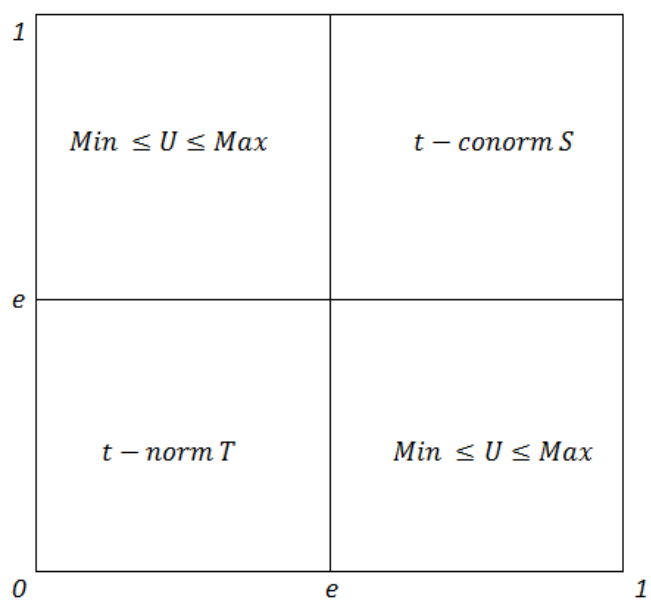

Fig. 2: General structure of a uninorm $U$ with neutral element $e$.

One of the best known families of uninorms is $U_{\text {min }}$ (see [12]). These uninorms, which are always conjunctive, are constructed from a t-norm $T$ and t-conorm $S$ using the minimum in the region $\mathrm{D}_{\mathrm{e}}$, i.e.,

$U_{\min }(x, y)= \begin{cases}e \cdot T\left(\frac{x}{e}, \frac{y}{e}\right) & \text { si } x, y \in[0, e] \\ e+(1-e) \cdot S\left(\frac{x-e}{1-e}, \frac{y-e}{1-e}\right) & \text { si } x, y \in[e, 1] \\ \min (x, y) & \text { si } x, y \in D_{e}\end{cases}$

Fig. 3 shows two uninorms belonging to the $U_{\min }$ class, where the minimum and algebraic product are taken as t-norms, and as t-conorms the associated maximum and algebraic sum, respectively.

In this study we consider a parameter $e$ determined for each rule, i.e. we will have as many parameters as rulers $\left(e_{i}\right)$, in order to have a local aggregation mechanism for each rule. Thus, we try to obtain the best results in accuracy as we obtained when we studied the adaptive t-norms $[4,5]$ previously. This model generalizes those based on t-norms [2] and t-conorms [6] when $e_{i}=1$ and $e_{i}=0$ respectively for every rules of the RB.

The use of uninorms as adaptive aggregation operator allows us to adapt the influence of the matching degree in a particular way. The effect of the parameters in the adaptive conjunction is as follows: The neutral element determines in each case whether the aggregation operator uninorm acts as t-norm (conjunction), as t-conorm (disjunction), or as another operator (area $D_{e}$ ).

The area $D_{e}$ of $U_{\min }$ also behaves as a conjunction operator. In addition, you can choose the t-norm and tconorm you want. Note that the use of conjunctive uninorms does not change the logical meaning of the infer- ence of the classical case. This is because these operators match with the classical conjunction when we restrict to $[0,1]^{2}$.

$$
\text { (a) } U_{\min }\left\{\begin{array}{c}
t-\text { norm }=T_{\text {Minimum }} \\
t-\text { conorm }=S_{\text {Maximum }}
\end{array}\right.
$$

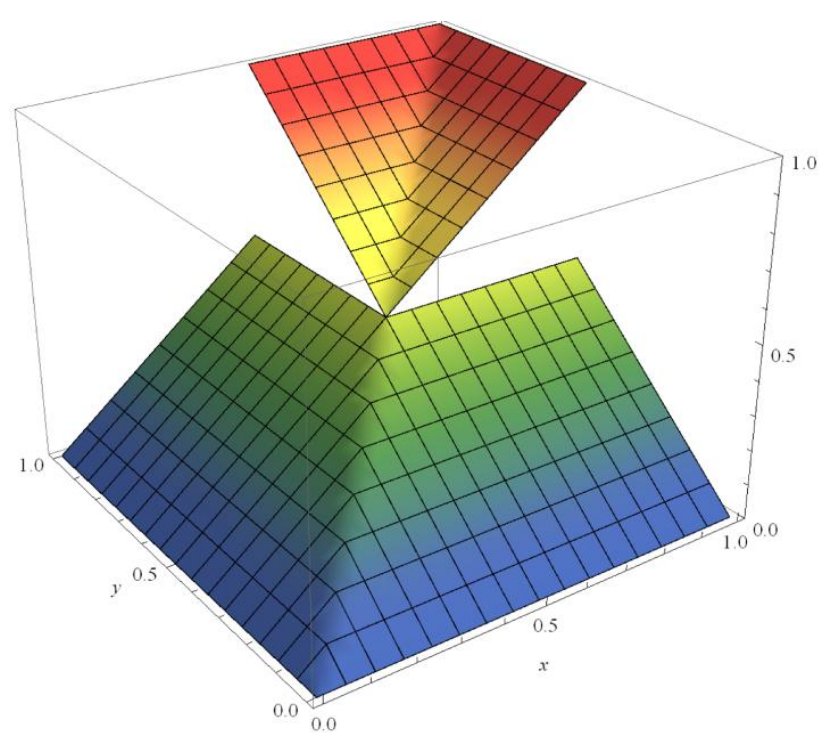

(b) $U_{\min }\left\{\begin{array}{c}t-\text { norm }=T_{\text {Algebraic }} \\ t-\text { conorm }=S_{\text {Algebraic }}\end{array}\right.$

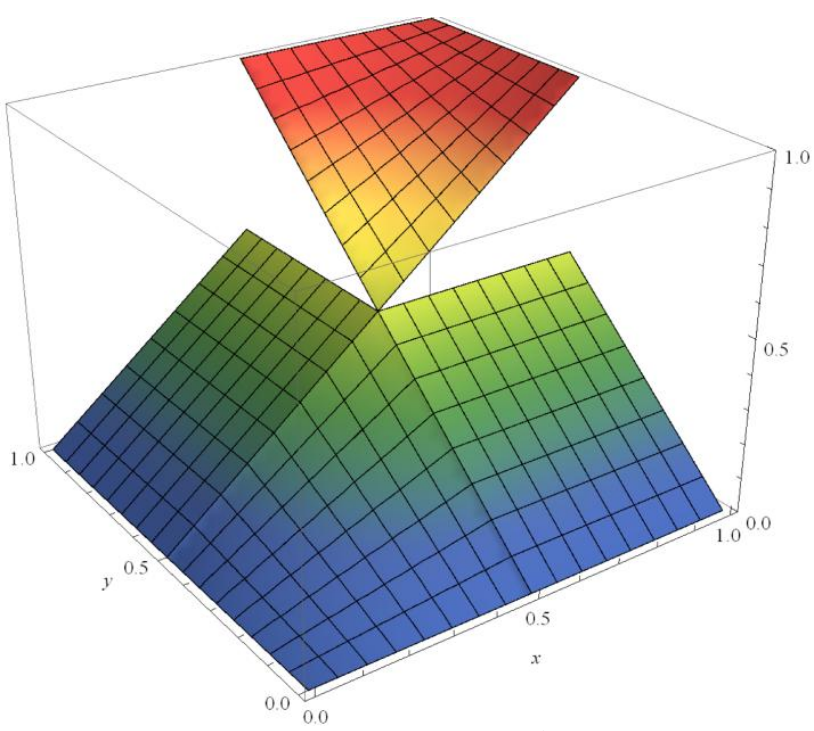

Fig.3: Uninorms of $U_{\min }$ considered with $e=\frac{1}{2}$.

When using uninorms, it should be noted that the aggregation of each rule behavior not only depends on the value of the $e_{i}$ parameter to be a conjunction of a disjunction, because it also depends on the value of the matching of the antecedents, i.e., the same specific value of the $e_{i}$ parameter could aggregate the antecedents of a rule as a conjunction or a disjunction also depending on the values of each input value. Therefore, it is not simply a mechanism that makes each rule always use conjunctive or disjunctive aggregation. Viewing Fig. 3, where $e=0.5$, the operator acts on the top of the 
chart as a conjunction for certain values of $x$ and $y$, and as a disjunction on the remainder.

\section{Evolutionary Learning of Uninorms for Infer- ence System}

In this section, we describe the evolutionary model we propose to learn the adaptive aggregation operators based on uninorms.

In our previous studies on adaptive conjunction operators based on parameterized t-norms, we used evolutionary algorithms with real coding $[4,5]$. They proved to be very suitable in this task, so we decided to use them again in this study. The evolutionary algorithm selected is a well known CHC type genetic algorithm [17]. Its goal is to learn the neutral element $e_{i}$ for each rule of the RB.

\subsection{Codification scheme and initial population}

We use a real coding scheme of size $m$ (with $m$ representing the number of rules of RB) in which the aggregation operator of the different rules is coded,

$$
A=\left(e_{1}, \ldots, e_{m}\right) \mid e_{i} \in[0,1] .
$$

Each gene $e_{i}$ represents the corresponding parameter that belongs to the $\mathrm{i}$-th rule. The initial population will be comprised of two different kind of individuals: A single one that has all the genes initially set to 1 in order to begin the evolutionary process with all the rules with the aggregation operator set as a t-norm (depending on the t-norm used to built the uninorm), and the remaining individuals randomly initialized within the interval $[0,1]$.

\subsection{Evaluation}

The fitness function used was the classical Mean Square Error (MSE) which measures the accuracy of the system.

$$
\operatorname{MSE}(F M)=\frac{\frac{1}{2} \sum_{k=1}^{P}\left(y_{k}-F M\left(x_{k}\right)\right)^{2}}{P}
$$

where FM denotes the fuzzy model whose Inference System uses the adaptive aggregation operator based on uninorms; the inference operator is the Minimum tnorm, and the defuzzification method is the Centre of Gravity weighted by the matching degree. This measure uses a set of system evaluation data formed by $P$ pairs of numerical data $Z_{k}=\left(x_{k}, y_{k}\right), k=1, \ldots, P$, with $x_{k}$ as the values of the input variables, and $y_{k}$ as the corresponding values of the associated output variables.

\subsection{Crossover operator}

Although the CHC evolutionary model was conceived for binary-coded problems, there are real-coded versions. In these cases, the BLX- $\alpha$ [18] crossover is used in order to recombine the parent's genes (we set $\alpha$ $=0.5$ ). This produces two descendents for each pair of parents, so the offspring generated by this crossover operator is of the same size as the initial population. The Hamming distance is computed by translating the realcoded genes into strings and by taking into account whether each character is different or not. Only those string pairs which differ from each other by some number of bits (mating threshold) are crossed.

The initial threshold is set to $\mathrm{L} / 4$, where $\mathrm{L}$ is the length of the string. When no offspring is inserted into the new population, the threshold is reduced by 1 .

\subsection{Restart}

No mutation is applied during the recombination phase. Instead, when the population converges or the search stops making progress (i.e., the difference threshold has dropped to zero and none of the new generated offspring are better than any member of the parent population), the population is reinitialized. The restarted population completely consists of random individuals except for one of them, which must be the best individual found so far.

\section{Experimental study and analysis of results}

The purpose of this work is to analyze and compare the practical behavior of various representative uninorms of the $U_{\min }$ family as adaptive aggregation operator. To do so, we selected 23 different problems with diverse complexities (different number of variables and patterns/data samples). Table 1 summarizes the main features of the different problems considered and their link to the KEEL dataset repository [19], and UCI Machine Learning Repository where they can be downloaded. Problems were selected from lower to higher complexity, covering a range from 2 to 16 input variables and from 43 to 20,640 examples.

This section is organized as follows:

- First, in Subsection 4.1 we describe the experimental setup and introduce and justify the different uninorms used.

- Second, Subsection 4.2 focuses on comparing and analyzing the result of the different uninorms.

\subsection{Experimental Setup}

This section describes the experimental setup, including a brief description of the methods and the nonparametric statistical tests considered for the performed comparisons.

To evaluate the effectiveness of the uninorms and establish a comparison, we built several adaptive Fuzzy Inference System models based on adaptive aggregation operators using the uninorms. Specifically, we chose a set of uninorms derived from $U_{\min }$ family. In this sense, we have selected all the classic t-norms and the associated t-conorm for each one.

The whole set of fuzzy models are illustrated in Table 2 . We have the evolutionary aggregation models, which are based on the linguistic RB learned with the WM-method, and later altering their parameterized connectors using quite a few combinations of several uninorms of $U_{\min }$. 


\begin{tabular}{lcccl}
\hline Problem & Abbr. & Inst. & Var. & Repo. \\
\hline Diabetes & DBT & 43 & 2 & KEEL \\
Electric-1 & ELE1 & 495 & 2 & KEEL \\
Plastic & PLA & 1650 & 2 & KEEL \\
Quake & QUA & 2178 & 3 & KEEL \\
Electric-2 & ELE2 & 1056 & 4 & KEEL \\
Laser & LAS & 993 & 4 & KEEL \\
AutoMPG6 & MP6 & 392 & 5 & KEEL \\
Friedman & FRI & 1200 & 5 & KEEL \\
Dee & DEE & 365 & 6 & KEEL \\
MachineCPU & CPU & 209 & 6 & KEEL \\
Anacalt & ANA & 4052 & 7 & KEEL \\
AutoMPG8 & MP8 & 392 & 7 & KEEL \\
Abalone & ABA & 4177 & 8 & KEEL \\
California & CAL & 20640 & 8 & KEEL \\
Concrete & CON & 1030 & 8 & KEEL \\
Stock & STP & 950 & 9 & KEEL \\
Weather Ankara & WAN & 1609 & 9 & KEEL \\
Weather Izmir & WIZ & 1461 & 9 & KEEL \\
Wine-red & WIR & 1599 & 11 & UCI \\
Forest Fires & FOR & 517 & 12 & KEEL \\
Mortgage & MOR & 1049 & 15 & KEEL \\
Treasury & TRE & 1049 & 15 & KEEL \\
Baseball & BAS & 337 & 16 & KEEL \\
\hline
\end{tabular}

Table 1: Datasets considered for the experimental study. They are available at KEEL and UCI repositories: (http://sci2s.ugr.es/keel/datasets.php)

(http://www.ics.uci.edu/ mlearn/MLSummary.html).

\begin{tabular}{cl}
\hline Method & \multicolumn{1}{c}{ Description } \\
\hline $\boldsymbol{M}\left(U_{T_{M}, S_{M}, e}\right)$ & Built with $\mathrm{T}_{\text {Minimum }} \mathrm{S}_{\text {Maximun }}$ (Fig. 3a) \\
$\boldsymbol{M}\left(U_{T_{H}, S_{H}, e}\right)$ & Built with $\mathrm{T}_{\text {Hamacher }} \mathrm{S}_{\text {Hamacher }}$ \\
$\boldsymbol{M}\left(U_{T_{A}, S_{A}, e}\right)$ & Built with $\mathrm{T}_{\text {Algebraic }} \mathrm{S}_{\text {Algebraic }}$ (Fig. 3b) \\
$\boldsymbol{M}\left(U_{T_{E}, S_{E}, e}\right)$ & Built with $\mathrm{T}_{\text {Einstein }} \mathrm{S}_{\text {Einstein }}$ \\
$\boldsymbol{M}\left(U_{T_{B}, S_{B}, e}\right)$ & Built with $\mathrm{T}_{\text {Bounded }} \mathrm{S}_{\text {Bounded }}$ \\
$\boldsymbol{M}\left(U_{T_{D}, S_{D}, e}\right)$ & Built with $\mathrm{T}_{\text {Drastic }} \mathrm{S}_{\text {Drastic }}$ \\
\hline
\end{tabular}

Table 2: Uninorms considered for comparison

The population size of the evolutionary algorithm was fixed to 61 individuals. The evolutionary algorithms performed 10000 evaluations. Concerning the Knowledge Bases, linguistic partitions were considered consisting of 3 triangular shaped linguistic terms for all problems.

Table 3 shows the average number of rules (\#R) and the MSE with training and test datasets of the FRBSs whose RBs were obtained with the well-known ad-hoc data driven algorithm of Wang and Mendel (WM) [20]. They are shown simply as a reference.

We considered a 5-fold cross-validation model, i.e. 5 random partitions of data each with $20 \%$, and the combination of four of them $(80 \%)$ as training and the remaining one as test. For each of the 5 data partitions, the evolutionary methods were run six times using different seeds for the random number generators, showing for each problem the averaged results of a total of 30 runs. To compare the different approaches, we focus on MSE for training and test. For each dataset, we compute the mean values over the 30 trials of the MSE on the training and test sets.

\begin{tabular}{|c|c|c|c|}
\hline Name & \#R & $\mathrm{MSE}_{\mathrm{TRA}}$ & $\mathrm{MSE}_{\mathrm{TST}}$ \\
\hline DBT & 15.8 & 0.16754 & 0.28898 \\
\hline ELE1 & 12.4 & 234712 & 242043 \\
\hline PLA & 14.8 & 3.43445 & 3.55722 \\
\hline QUA & 53.6 & 0.02582 & 0.02666 \\
\hline ELE2 & 65 & 56135 & 56359 \\
\hline LAS & 58.4 & 132.60 & 139.28 \\
\hline MP6 & 116 & 4.338 & 6.819 \\
\hline FRI & 766.2 & 1.458 & 2.532 \\
\hline DEE & 178.4 & 0.07059 & 0.11444 \\
\hline CPU & 41 & 1134 & 3859 \\
\hline ANA & 123.6 & 0.02703 & 0.03009 \\
\hline MP8 & 161.4 & 4.142 & 7.178 \\
\hline $\mathrm{ABA}$ & 199 & 3.341 & 3.476 \\
\hline CAL & 623.8 & 38.3230 & 38.7122 \\
\hline $\mathrm{CON}$ & 309.8 & 35.3 & 47.6 \\
\hline STP & 265.4 & 1.452 & 1.487 \\
\hline WAN & 456.8 & 4.878 & 6.128 \\
\hline WIZ & 399.2 & 3.107 & 4.007 \\
\hline WIR & 714.2 & 0.22936 & 0.25095 \\
\hline FOR & 374.6 & 1435 & 34234 \\
\hline MOR & 198.8 & 0.12812 & 0.13387 \\
\hline TRE & 196 & 0.40155 & 0.40529 \\
\hline BAS & 252.6 & 78213 & 648520 \\
\hline
\end{tabular}

Table 3: Reference values of average number of rules and MSE of the FRBSs, with Minimum t-norm aggregation. Values of MSE in this table must be multiplied by $10^{8}$ in the case of CAL.

To assess whether there are significant differences among the results, we use statistical analysis [21, 22] and in particular non-parametric tests. We employ different approaches for multiple comparisons, including Friedman's test [23], to detect statistical differences among a group of results, and the Finner post-hoc test [24] to observe the difference in performance between the methods and the retention or rejection of the hypothesis with the level of significance fixed. To perform the tests, we used a level of confidence $\alpha=0.05$. In particular, these tests are based on computing the differences on sample means (typically, mean test errors obtained by a pair of different algorithms on different datasets). In the classification framework, these differences are well defined, as the errors are in the same domain. In the regression framework, to make the differences comparable, we adopt a normalized difference DIFF, defined as:

$$
D I F F=\frac{\text { Mean }(\text { other })-\text { Mean }(\text { reference })}{\text { Mean }(\text { other })}
$$

where Mean(x) represents the MSE obtained by the $\mathrm{x}$ algorithm. This difference expresses the improvement percentage of the reference algorithm on the other one.

\subsection{Results and Analysis}

The results obtained by the different FRBSs are shown in Table 4. This table is grouped in columns by FRBSs models based on the different uninorms to be compared and shows the average of the results obtained by each model for all the datasets employed. For each one, the 
columns show the average MSE for training and test datasets, respectively. We have highlighted in bold the best accuracies for test.

Table 5 shows the rankings obtained with the Friedman's test. They tell us that there are significant differences among the results observed with all datasets except one when the $\mathrm{p}$-Fried $<0.05$. Indeed, there are significant differences in MSE $\mathrm{TST}_{\text {. The }}$. Thest ranking is obtained by $\boldsymbol{M}\left(U_{T_{A}, S_{A}, e}\right)$. However, we can point out that $\boldsymbol{M}\left(U_{T_{E}, S_{E}, e}\right)$ is very close.

Finally, Table 6 shows the results when using Finner's post-hoc procedure to compare the best ranking model in each case with the remaining ones. Algorithms are ordered by the p-value. Indeed, the Finner test rejects the hypothesis of equality for $\mathrm{MSE}_{\mathrm{TST}}$ when the control algorithm is $\boldsymbol{M}\left(U_{T_{A}, S_{A}, e}\right)$ except for $\boldsymbol{M}\left(U_{T_{E}, S_{E}, e}\right)$.

Therefore, analyzing the results from Table 4 , and the statistical evidence obtained on Tables 5 and 6 , we can highlight that:

- The model $\boldsymbol{M}\left(U_{T_{A}, S_{A}, e}\right)$ achieves the best accuracy with statistical significance compared to the remaining models, except with $\boldsymbol{M}\left(U_{T_{E}, S_{E}, e}\right)$, which shows the second best result of the study. Thus, they are the two most recommended uninorms for use in linguistic fuzzy modeling in practice.

- The worse results were obtained by $\boldsymbol{M}\left(U_{T_{D}, S_{D}, e}\right)$ and $\boldsymbol{M}\left(U_{T_{H}, S_{H}, e}\right)$, surely because they come from the Drastic and Hamacher connectives, which were the worst in the conjunction comparative study carried out in [1].

- We can also point out that the uninorm that comes from the Minimum t-norm and Maximum t-conorm is not among the better results obtained as might be expected.

\section{Conclusions and Future Works}

In this work we have studied and compared the use of different uninorms of $U_{\text {min }}$ family for linguistic fuzzy modeling. We have proposed an evolutionary learning model where the aggregation connector parameters are learnt, performing an Evolutionary Adaptive Inference System based on an aggregation operator using uninorms.

The flexibility of the parameterized antecedent aggregation based on uninorms improves the accuracy of linguistic fuzzy models. We compared the accuracy of six models built with uninorms employed with 23 different application, and performed a statistical study. We found that the uninorms based on the Algebraic and Einstein product and their associated t-conorm are the ones that show the best mean accuracy.

Finally, we are extending the study, increasing the number of uninorms and using other parameterized connectives as adaptive t-norms.

\begin{tabular}{cc}
\hline Method & $\begin{array}{c}\text { Ranking on MSE } \\
\text { (p-value Fried: 4.249E-8) }\end{array}$ \\
\hline $\boldsymbol{M}\left(U_{T_{M}, S_{M}, e}\right)$ & 3.56521 \\
$\boldsymbol{M}\left(U_{T_{H}, S_{H}, e}\right)$ & 4.47826 \\
$\boldsymbol{M}\left(U_{T_{A}, S_{A}, e}\right)$ & 2.17391 \\
$\boldsymbol{M}\left(U_{T_{E}, S_{E}, e}\right)$ & 2.21739 \\
$\boldsymbol{M}\left(U_{T_{B}, S_{B}, e}\right)$ & 3.60869 \\
$\boldsymbol{M}\left(U_{T_{D}, S_{D}, e}\right)$ & 4.95652 \\
\hline
\end{tabular}

Table 5: Rankings obtained with Friedman's test on $\mathrm{MSE}_{\mathrm{TST}}$.

\begin{tabular}{|c|c|c|c|c|c|c|c|c|c|c|c|c|}
\hline \multirow[t]{2}{*}{ Datasets } & \multicolumn{2}{|c|}{$\boldsymbol{M}\left(U_{T_{M}, S_{M}, e}\right)$} & \multicolumn{2}{|c|}{$\boldsymbol{M}\left(U_{T_{H}, S_{H}, e}\right)$} & \multicolumn{2}{|c|}{$\boldsymbol{M}\left(U_{T_{A}, S_{A}, e}\right)$} & \multicolumn{2}{|c|}{$\boldsymbol{M}\left(U_{T_{E}, S_{E}, e}\right)$} & \multicolumn{2}{|c|}{$\boldsymbol{M}\left(U_{T_{B}, S_{B}, e}\right)$} & \multicolumn{2}{|c|}{$\boldsymbol{M}\left(U_{T_{D}, S_{D}, e}\right)$} \\
\hline & $\mathrm{MSE}_{\mathrm{TRA}}$ & $\mathrm{MSE}_{\mathrm{TST}}$ & $\mathrm{MSE}_{\mathrm{TRA}}$ & $\mathrm{MSE}_{\mathrm{TST}}$ & $\mathrm{MSE}_{\mathrm{TRA}}$ & $\mathrm{MSE}_{\mathrm{TST}}$ & $\mathrm{MSE}_{\mathrm{TRA}}$ & $\mathrm{MSE}_{\mathrm{TST}}$ & $\mathrm{MSE}_{\mathrm{TRA}}$ & $\mathrm{MSE}_{\mathrm{TST}}$ & $\mathrm{MSE}_{\mathrm{TRA}}$ & $\mathrm{MSE}_{\mathrm{TST}}$ \\
\hline DBT & 0.10823 & 0.26949 & 0.10337 & 0.27501 & 0.09911 & 0.28248 & 0.09712 & 0.28925 & 0.09129 & 0.29752 & 0.07062 & 0.3322 \\
\hline ELE1 & 201347 & 222140 & 198936 & 217899 & 194222 & 212767 & 192269 & 210907 & 186487 & 211457 & 186252 & 231996 \\
\hline PLA & 1.94901 & 2.04138 & 3.1608 & 2.89966 & 1.77626 & 1.86015 & 1.75806 & 1.84078 & 1.7357 & 1.81337 & 1.89159 & 1.97871 \\
\hline QUA & 0.02166 & 0.02284 & 0.02129 & 0.02244 & 0.02117 & 0.02254 & 0.02093 & 0.02242 & 0.02067 & 0.02243 & 0.02014 & 0.02231 \\
\hline ELE2 & 38664 & 41356 & 55418 & 55531 & 31695 & 34704 & 32195 & 35973 & 36525 & 42534 & 41958 & 48857 \\
\hline LAS & 80.47 & 100.52 & 68.34 & 76.40 & 45.19 & 61.10 & 42.86 & 60.38 & 42.74 & 62.55 & 43.51 & 71.67 \\
\hline MP6 & 2.871 & 6.049 & 4.214 & 3.648 & 2.429 & 5.790 & 2.348 & 5.899 & 2.228 & 6.253 & 2.201 & 6.309 \\
\hline FRI & 1.138 & 2.484 & 0.996 & 2.407 & 0.938 & 2.371 & 0.931 & 2.488 & 0.965 & 3.091 & 1.140 & 3.527 \\
\hline DEE & 0.04941 & 0.10866 & 0.04956 & 0.11055 & 0.03973 & 0.11356 & 0.03724 & 0.11824 & 0.03359 & 0.13019 & 0.03301 & 0.14142 \\
\hline $\mathrm{CPU}$ & 866 & 3600 & 1112 & 3816 & 744 & 3445 & 751 & 3465 & 739 & 4917 & 682 & 4990 \\
\hline ANA & 0.00763 & 0.01093 & 0.02697 & 0.03004 & 0.00758 & 0.01098 & 0.00756 & 0.01097 & 0.00756 & 0.01112 & 0.00754 & 0.01127 \\
\hline MP8 & 2.765 & 6.685 & 4.185 & 7.158 & 2.302 & 6.382 & 2.207 & 6.407 & 2.048 & 6.740 & 2.132 & 6.840 \\
\hline $\mathrm{ABA}$ & 2.754 & 2.967 & 3.293 & 3.421 & 2.458 & 2.720 & 2.408 & 2.692 & 2.370 & 2.694 & 2.403 & 2.779 \\
\hline CAL & 27.7969 & 28.4289 & 37.5960 & 37.9909 & 23.5117 & 24.4011 & 22.9556 & 23.8884 & 22.7064 & 23.7248 & 23.2553 & 24.3343 \\
\hline $\mathrm{CON}$ & 23.7 & 40.8 & 35.8 & 47.5 & 18.4 & 36.32 & 17.6 & 36.37 & 17.5 & 38.5 & 20.4 & 41.5 \\
\hline STP & 0.880 & 1.011 & 0.760 & 0.868 & 0.562 & 0.694 & 0.547 & 0.690 & 0.553 & 0.709 & 0.584 & 0.758 \\
\hline WAN & 2.933 & 4.682 & 4.700 & 5.907 & 2.454 & 4.338 & 2.512 & 4.507 & 2.630 & 5.313 & 2.932 & 5.959 \\
\hline WIZ & 1.840 & 2.985 & 2.958 & 3.839 & 1.417 & 2.603 & 1.471 & 2.665 & 1.601 & 3.072 & 1.820 & 3.777 \\
\hline WIR & 0.19185 & 0.22784 & 0.20948 & 0.22976 & 0.15967 & 0.21693 & 0.15503 & 0.21508 & 0.14891 & 0.2211 & 0.14865 & 0.23139 \\
\hline FOR & 1242 & 19743 & 1460 & 19800 & 965 & 19783 & 949 & 20149 & 1017 & 28175 & 1057 & 31042 \\
\hline MOR & 0.05496 & 0.06695 & 0.1263 & 0.11212 & 0.04939 & 0.06426 & 0.04986 & 0.06443 & 0.05099 & 0.06764 & 0.05806 & 0.07466 \\
\hline TRE & 0.15922 & 0.17671 & 0.365 & 0.24639 & 0.11451 & 0.13932 & 0.1152 & 0.14198 & 0.11719 & 0.14512 & 0.12356 & 0.16089 \\
\hline BAS & 59739 & 620092 & 84197 & 617578 & 49115 & 635217 & 49122 & 639570 & 51942 & 750424 & 54379 & 809707 \\
\hline
\end{tabular}

Table 4: Average results of the six different adaptive conjunction FRBS models based on Uninorms. Results in this table for MSE must be multiplied by $10^{8}$ in the case of CAL. 


\begin{tabular}{cccc}
\hline I & Method & pFinner & Hypot \\
\hline 1 & $\boldsymbol{M}\left(U_{T_{D}, S_{D}, e}\right)$ & 2.1688389 E-6 & Reject. \\
2 & $\boldsymbol{M}\left(U_{T_{H}, S_{H}, e}\right)$ & $1.1266681 \mathrm{E}-4$ & Reject. \\
3 & $\boldsymbol{M}\left(U_{T_{B}, S_{B}, e}\right)$ & 0.023341040 & Reject. \\
4 & $\boldsymbol{M}\left(U_{T_{M}, S_{M}, e}\right)$ & 0.023341040 & Reject \\
5 & $\boldsymbol{M}\left(U_{T_{\mathrm{E}}, S_{E}, e}\right)$ & 0.937182922 & Accept \\
\hline
\end{tabular}

Table 6: Finner's test table with p-value $=0.05$ for the methods on $\mathrm{MSE}_{\mathrm{TST}}$ on accuracy. Control: $\boldsymbol{M}\left(U_{T_{A}, S_{A}, e}\right)$.

\section{References}

[1] O. Cordón, F. Herrera and A. Peregrín, Applicability of the fuzzy operators in the design of fuzzy logic controllers, Fuzzy Sets and Systems 86:1541, 1997.

[2] O. Cordón F. Herrera and A. Peregrín, Searching for basic properties obtaining robust implication operators in fuzzy control, Fuzzy Sets and Systems, 111: 237-251, 2000.

[3] F. Herrera, Genetic fuzzy systems: taxonomy, current research trends and prospects, Evolutionary Intelligence, 1: 27-46, 2008.

[4] J. Alcala-Fdez, F. Herrera, F.A. Márquez and A. Peregrín, Increasing fuzzy rules cooperation based on evolutionary adaptive inference systems, International Journal of Intelligent System, 22 (9): 1035-1064, 2007.

[5] F.A. Márquez, A. Peregrín and F. Herrera, Cooperative evolutionary learning of fuzzy rules and parametric aggregation connectors for Mamdani linguistic fuzzy systems, IEEE Transactions on Fuzzy System 15 (6): 1162-1178, 2007.

[6] M. Mizumoto, Pictorial representations of fuzzy connectives, part I: Cases of T-norms, T-conorms and averaging operators, Fuzzy Sets System., 3: 217-242, 1989.

[7] I.Z. Batyrshin, I.J. Rudas, L.A. Villa and P. Cortés, On the monotone sum of basic tnorms in the construction of parametric families of digital conjunctors for fuzzy systems with reconfigurable logic, Knowledge-Based Systems, Special Issue on Advances in Fuzzy Knowledge Systems: Theory and Application 38: 27-36, 2013.

[8] H. Wu and J.M. Mendel, On choosing models for linguistic connector words for Mamdani fuzzy logic systems, IEEE Transactions on Fuzzy Systems 4: 29-44, 2004.

[9] J. Fodor, I. J. Rudas and B. Bede, Uninorms and absorbing norms with applications to image processing, in proceedings of 4th Serbian-Hungarian Joint Symposium on Intelligent Systems, SYS 2006, 59-72, 2006.

[10] G. Gosztolya and J. Dombi, Applying Representative Uninorms for phonetic classifier combination, in proceedings of 11th International Conference Modeling Decisions for Artificial Intelligence, MDAI 2014, Lectures Notes in Computer Science 8825, pages 182-191, Springer-Verlag, 2014.

[11] R. R. Yager and A. Rybalov. Uninorm aggregation operators, Fuzzy Sets and Systems, 80: 111120, 1996.
[12] J. C. Fodor, R. R. Yager, and A. Rybalov: Structure of uninorms. Proceedings of the International Journal of Uncertainty, Fuzziness and Knowledgebased Systems 5: 411-127, 1997.

[13] E. Trillas and L. Valverde, On implication and indistiguishability in the setting of fuzzy logic. In Management Decision Support Systems Using Fuzzy Sets and Possibility Theory, J. Kacpryzk and R. Yager, Eds. New York: Verlag TüV Rheinland, , 198-212, 1985

[14] M. Gupta and J. Qi, Theory of T-norms and fuzzy inference methods, Fuzzy Sets and Systems, 40: 431-450, 1991.

[15] I.J. Rudas E. Pap and J. Fodor, Information aggregation in intelligent systems: an application oriented approach, Knowledge-Based Systems, Special Issue on Advances in Fuzzy Knowledge Systems: Theory and Application 38 (2013) 3-13.

[16] J. Fodor and T. Keresztfalvi, Nonstandard Conjunctions and Implications in Fuzzy Logic, International Journal of Approximate Reasoning 12: 69-84, 1995.

[17] L.J. Eshelman, The CHC Adaptive search algorithm: How to have safe search when engaging in nontradi-tional genetic recombination. Foundations of Genetic Algorithms1,Rawlins,G.J.E.(Eds), 265-283, 1991.

[18] F. Herrera, M. Lozano and A. Sanchez, A taxonomy for the crossover operator for real-coded genetic algo-rithms: an experimental study, International Journal of Intelligent System 18: 309-338, 2003.

[19] J. Alcala-Fdez, L. Sanchez, S. Garcia, M. del Jesus, S. Ventura, J. Garrell, J. Otero, C. Romero, J. Bacardit, V. Rivas, J. Fernandez and F. Herrera, Keel: a software tool to assess evolutionary algorithms for data mining problems, Soft Computing 13(3): 307-318, 2009.

[20] L. X. Wang and J.M. Mendel, Generating fuzzy rules by learning from examples, IEEE Transactions on Systems, Man, and Cybernetics, 22(6), 1414-1427, 1992.

[21] J. Demšar, Statistical comparisons of classifiers over multiple data sets, Journal of Machine Learning Research, 7: 1-30, 2006.

[22] S. García, A. Fernández, J. Luengo and F. Herrera, Advanced nonparametric tests for multiple comparisons in the design of experiments in computational intelligence and data mining: experimental analysis of power, Information Sciences 180(10): 2044-2064, 2010.

[23] M. Friedman, The use of ranks to avoid the assumption of normality implicit in the analysis of variance, Journal of the American Statistical Association, 32:674-701, 1937.

[24] H. Finner, On a monotonicity problem in stepdown multiple test procedures, Journal of the American Statistical Association, 88 (1993) 920 923. 